\title{
Difficult management of a patient with pr10 related anaphylaxis
}

Eleonora Nucera, Arianna Aruanno, Alessandro Buonomo, Simona Mezzacappa, Angela Rizzi, Domenico Schiavino

Allergy Unit, Fondazione Universitaria - Policlinico “A. Gemelli”, Università Cattolica del Sacro Cuore, Rome, Italy

Adv Dermatol Allergol 2016; XXXIII (5): 386-388

DOI: $10.5114 / a d a .2016 .62847$

Proteins belonging to pathogenesis-related (PR10) ones consist in Bet $v 1$ homologues, proteins that have an aminoacid sequence homology with the allergen Bet $v 1$ from birch pollen [1].

Bet $v 1$ is the major allergen of birch and about $50-70 \%$ of birch pollen allergic patients, usually after respiratory sensitization, report symptoms after ingestion of fruits and vegetables, such as tree nuts, kiwi, soybean and those belonging to the Rosaceae family (apple, pear, peach, plum, apricot, almond) and Apiaceae family (celery, carrot, fennel). Other Bet v 1 homologues include Mal d 1 from apple, Pru $p 1$ from peach, Pru a v 1 from cherry, Pru a $r 2$ from apricot, Pyr c 1 from pear, Api g 1 from celery, Dau c 1 from carrot and Cor a 1 from hazelnut [2].

Many proteins homologous to Bet $v 1$, in particular those present in the fruits of the Rosaceae family, are extremely labile and easily degraded by heat, oxidation, procedures of extraction, pepsin digestion and they are randomly distributed in pulp and peel [3, 4]. For this reason the skin prick tests with commercial extracts of foods are less specific than those with fresh fruits and vegetables, and remain the best method for the diagnosis of food allergy in these patients [5].

Therefore, PR10 allergic patients mostly experience local reactions in the oropharyngeal mucosa (oral allergy syndrome - OAS: oral itching, swelling of the lips, tongue and throat, hoarseness and laryngeal oedema) after eating fresh foods, while they usually tolerate heatprocessed foods such as commercial fruit juices.

In the literature there are few articles about PR-10 related proteins induced anaphylaxis [6-8].

We describe the case of a 30-year-old girl with perennial rhinoconjunctivitis and asthma with an aggravation from February to June, who referred OAS after the ingestion of apricot, cherry and strawberry, and throat constriction, generalized urticaria, lip and palpebral oedema after eating apple, kiwi, peach, pear, pepper and juice with carrots, lemon and orange.

We performed an allergological evaluation including skin prick tests (SPT) to aeroallergens, latex, vegetables and panallergens (apple PR10 $50 \mu \mathrm{g} / \mathrm{ml}$, peach Lipid Transfer Protein $50 \mu \mathrm{g} / \mathrm{ml}$ and palm profiling $50 \mu \mathrm{g} / \mathrm{ml}$ ) (Alk-Abellò, Milan, Italy) and assay of specific IgE (UniCAP, Phadia, Uppsala, Sweden) for the positive allergens. Moreover, the culprit vegetables have been investigated by the prick by prick method.

The patient had positive SPT to inhalant allergens (dermatophaghoides, birch tree, cypress, plane, hazel tree), apple, peach, pear, kiwi, almond and hazelnut, birch and apple PR10.

The specific IgEs were positive to dermatophaghoides (23 kU/l), birch (13.4 kU/l), hazel $(6.39 \mathrm{kU} / \mathrm{l})$, peach (1.74 $\mathrm{kU} / \mathrm{l})$, pear $(3.06 \mathrm{kU} / \mathrm{l})$, rPru p $1(1.42 \mathrm{kU} / \mathrm{l})$ and rBet $\vee 1$ $(14 \mathrm{kU} / \mathrm{l})$.

So to confirm these results, we also performed the Mal d 1 basophil activation test that resulted positive. Then, she underwent a double-blind placebo-control food challenge (DBPCFC) with Golden apple without peel, which resulted positive (bronchospasm, rhinitis and conjunctivitis) at $10 \mathrm{~g}$ of apple; these symptoms receded with aerosol therapy (salbutamol and beclomethasone) and intravenous treatment (methylprednisolone and chlorpheniramine). Double-blind placebo-control food challenges with other culprit fruits were not performed because the patient had had recent reactions.

The current treatment of this allergy is still based on the avoidance of the offending foods. Our patient referred that avoidance of the most common food allergens was difficult and induced severe dietary restrictions with consequent metabolic imbalance. For these reasons,

Address for correspondence: Dr. Arianna Aruanno, Allergy Unit, Università Cattolica del Sacro Cuore, Policlinico “A. Gemelli”, Largo F. Vito, 1 - 00168 Rome, Italy, e-mail: aarianna@hotmail.it

Received: 30.12.2015, accepted: 7.02.2016. 
we tried to perform an oral desensitization treatment with a mix of the most consumed 4 apple cultivar (Stark, Golden, Fuji, Smith) in Italy on the basis of a previously published protocol $[9,10]$.

The treatment was interrupted for the onset of severe side effects after 2 months (recurrent asthma after the ingestion of the apples).

Because in the literature other authors described some attempts to PR-10 desensitization through birch immunotherapy [11-16] and one study showed [17] that such effect was rather long lasting, we performed a birch sublingual immunotherapy (Staloral 300 IR) to our patient. After 4 years of treatment the patient resolved completely her seasonal respiratory disease and we decided to evaluate the benefits on food allergy by DBPCFCs with the culprit foods. During the challenge test, the girl presented an anaphylactic reaction characterized by cough, bronchospasm, throat ache, lips and uvula oedema, conjunctivitis and abdominal pain after $40 \mathrm{gr}$ of pear without peel that receded completely after aerosol therapy (epinephrine, salbutamol and beclomethasone), intramuscular treatment with epinephrine and intravenous therapy (ranitidine, methylprednisolone and chlorpheniramine).

In the literature we found few cases of PR-10 related proteins induced anaphylaxis. In the first report the authors described the case of a patient with history of anaphylaxis induced by hazelnuts and peaches, but in this case the allergological work-up showed a double sensitization to proteins in both PR 10 (e.g. Bet v1, Cor a1, Pru p1) and LTP14 (e.g. Pru p3, Cor a8) [8]. In another study [6], the authors reported that when drinks containing PR-10 proteins rapidly consumed, for example during sporting activities, large concentrations of allergens can be reached without any intraoral symptoms and then lead to anaphylaxis. This phenomenon has often been described for soy milk, while in this case it occurred during exercise after the ingestion of an apple drink with $60 \%$ fruit concentration. In the last article, other cases of anaphylaxis were described after the ingestion of soy-containing dietary food products in patients allergic to birch pollen [7].

In this case report instead, we described another case of anaphylaxis caused by an apparently harmless panallergen that usually determined OAS after DBPCFC with fresh pear.

In patients as in our case where the desensitization treatment to PR-10 or birch does not work, the only therapeutic solution is the elimination diet of all the culprit foods with the risk to develop metabolic imbalance.

Bolhaar et al. [18] obtained a mutant of the major apple allergen Mal d 1 (Mald1 mut) whose hypo-allergenicity was demonstrated by the DBPCFC: in fact patients stimulated with Mal d 1 mut reacted at a higher dose than when stimulated with the classic Mal d 1. These observations lead to the hypothesis that patients with severe allergic reactions may benefit in the future from immunotherapy safer through the use of recombinant allergen.
Our case report emphasizes that not only well-known dangerous panallergens (nsLTP, storage proteins), but also apparently harmless proteins can be responsible for serious allergic reactions.

Therefore, we think that attending physicians should be aware of the possibility of serious allergic reactions and should be cautious in management of such patients.

\section{Acknowledgments}

Eleonora Nucera, Arianna Aruanno, Angela Rizzi, and Domenico Schiavino contributed equally to this work.

\section{Conflict of interest}

The authors declare no conflict of interest.

\section{References}

1. Hoffmann-Sommergruber K. Plant allergens and pathogenesis-related proteins. What do they have in common? Int Arch Allergy Immunol 2000; 122: 155-66.

2. Fernandez-Rivas M, Gonzales-Mancebo E, Rodriguez Perez R, et al. Clinically relevant peach lipid transfer protein, Pru $p$ 3, in the Spanish population. J Allergy Immunol 2003; 112: 789-95.

3. Ahrazem O, Jimeno L, López-Torrejón G, et al. Assessing allergen levels in peach and nectarine cultivars. Ann Allergy Asthma Immunol 2007; 99: 42-7.

4. Rudeschko O, Fahlbusch B, Henzgen M, et al. Investigation of the stability of apple allergen extracts. Allergy 1995; 50: 575-9.

5. Rodrigues-Alves R, Lopez A, Pereira-Santos MC, et al. Clinical, anamnestic and serological features of peach allergy in Portugal. Int Arch Allergy Immunol 2008; 149: 65-73.

6. Röseler S, Balakirski G, Plange J, et al. Anaphylaxis to PR-10 proteins (Bet v1 homologues). Hautarzt 2013; 64: 890-2.

7. Kleine-Tebbe J, Vogel L, Crowell DN, et al. Severe oral allergy syndrome and anaphylactic reactions caused by a Bet $\vee 1$ related PR-10 protein in soybean, SAM22. J Allergy Clin Immunol 2002; 110: 797-804.

8. Vanstreels L, Balakirski G, Röseler S, et al. Double sensitization to PR10 and PR-14 proteins. Hautarzt 2014; 65: 1017-8.

9. Patriarca G, Nucera E, Roncallo C, et al. Oral desensitizing treatment in food allergy: clinical and immunological results. Aliment Pharmacol Ther 2003; 17: 459-65.

10. Nucera E, Aruanno A, Lombardo C, et al. Apple desensitization in two patients with PR-10 proteins allergy. Allergy 2010; 65: 1060-1.

11. Kazemi-Shirazi L, Pauli G, Purohit A, et al. Quantitative IgE inhibition experiments with purified recombinat allergens indicate pollen-derived allergens as the sensitizing agents responsible for many forms of plant food allergy. J Allergy Clin Immunol 2000; 105: 116-25.

12. Asero R. Effects of birch pollen-specific immunotherapy on apple allergy in birch pollen-hypersensitivity patients. Clin Exp Allergy 1998; 28: 1368-73.

13. Modrzynski M, Zawisza E, Rapiejko P, et al. Specific-pollen immunotherapy in the treatment of oral allergy syndrome in patients with tree pollen hypersensitivity. Przegl Lek 2002; 59: 1007-10. 
14. Hermann D, Henzgen M, Frank E, et al. Effect of hyposensitization for tree pollinosis on associated apple allergy. I Invest Allergol Clin Immunol 1995; 5: 259-67.

15. Bolhaar ST, Tiemessen MM, Zuidmeer L, et al. Efficacy of birch-pollen immunotherapy on cross-reactive food allergy confirmed by skin tests and double-blind food challenges. Clin Exp Allergy 2004; 34: 761-9.

16. Bucher X, Pichler WJ, Dahinden CA, et al. Effect of tree pollen specific, subcutaneous immunotherapy on the oral allergy syndrome to apple and hazelnut. Allergy 2004; 59: 1272-6.

17. Asero R. How long does the effect of birch pollen injection SIT on apple allergy last? Allergy 2003; 58: 435-8.

18. Bolhaar ST, Zuidmeer L, Ma Y, et al. A mutant of the major apple allergen, Mal d 1, demonstrating hypoallergenicity in the target organ by double-blind placebo-controlled food challenge. Clin Exp Allergy 2005; 35: 1638-44. 